\title{
Treatment of dyslipidemia in diabetes. Should the lipid-management protocol which the ADA 2015 guideline recommends be used for Japanese as a reference?
}

\author{
Kazuhisa Tsukamoto ${ }^{1}$
}

Received: 12 July 2015/Published online: 7 August 2015

(C) The Japan Diabetes Society 2015

In January 2015, the American Diabetes Association (ADA) renewed the management guidelines for diabetic patients [1]. Within this renewal, a major revision was that for dyslipidemia treatment [2]. Recommended strategies for management of dyslipidemia in this revision were:

high-intensity statin therapy for patients with overt cardiovascular disease (CVD) and patients aged 40-75 years with CVD risk factors;

moderate-intensity statin therapy for patients aged 40 years or greater without CVD risk factors; and moderate or high-intensity statin therapy for patients under 40 or over 75 years of age with CVD risk factors.

Thus, this recommendation is completely different from previous ADA guidelines and from those of other countries that defined the target lipid levels for the treatment.

\section{Self-contradiction might exist in the ACC/AHA 2013 guideline}

This ADA guideline followed the concept of the 2013 American College of Cardiology/American Heart Association (ACC/AHA) guideline [3], which had been settled in accordance with "the 2011 report of the Institute of Medicine on the development of trustworthy clinical guidelines" [4]. Briefly, the expert panels had asked critical questions,

Kazuhisa Tsukamoto

kazut@fmu.ac.jp

1 Department of Metabolism, Diabetes and Nephrology, Aizu Medical Center, Fukushima Medical University, 21-2 Maeda, Tanisawa, Kawahigashi, Aizu-Wakamatsu, Fukushima 969-3492, Japan chosen and reviewed high-quality randomized controlled trials (RCTs) and reliable meta-analyses, and resolved the critical questions. Their precise analyses had identified "four statin benefit groups" which would benefit from management with statins, and diabetic patients were included among these groups. This identification of "four statin benefit groups" is a great advance in that physicians can easily identify patients requiring treatment for dyslipidemia. Besides this identification, the panels recommended administration of a fixed dose of the appropriate statin, leaving behind the "treat-to-target" protocol in which titration of agents is performed to achieve target lipid levels. The theoretical framework for this recommendation was:

fixed doses of specific statins were used in the analyzed RCTs; and these RCTs did not utilize protocols in which agent dose titration was performed to target specific LDL-C or nonHDL-C levels.

However, a pitfall could underlie this analytical process. That is to say, the critical questions on "whether target levels can be found for the treatment of dyslipidemia" are selfcontradictory. Most high-quality RCTs are conducted double-blind with specific agents at fixed doses. In other words, it is impossible to set different target lipid levels for each research group if trials are double-blind. Thus, no matter how we looked for RCTs which settled target lipid levels, it is theoretically impossible to find them in double-blind trials.

\section{Monitoring of lipid levels}

The ACC/AHA guideline recommends monitoring lipid levels periodically after initiation of statins. However, contrary to the "treat-to-target" protocol, the guideline 
insists that the values obtained should be used solely for assessing a patient's adherence to the medication and lifestyle modification, but not for titration of statin dose; the 2015 ADA guideline also follows this principle. The concept which underlies this principle is that "with high or moderate intensity statin therapy, more than $50 \%$ or around 30-50 \% LDL-C reduction, respectively, should be attained. Thus, titration of statin dose is not necessary". This principle, which is substantially identical to the "fireand-forget" approach, is very simple to use in the clinical setting. However, as we physicians know in daily clinical work, and as was recently reported in the literature [5], response to statin therapy varies among individuals, and those who do not respond well gain little benefit [5]. Furthermore, assessment of specific percentage reduction from the baseline level is, somewhat, a cause of concern; the absolute target LDL-C values used vary among patients, because of different initial lipid levels before treatment. In addition, because the general rate of LDL-C reduction with statins is greater for Japanese patients than for American patients, the statin dose recommended in the ACC/AHA guideline cannot be readily applicable for Japanese.

\section{Residual risks and non-statin agents}

Management for residual risks has received worldwide attention with the purpose of achieving a further reduction in atherosclerotic diseases. Among these risks, elevated residual cholesterol, low HDL-C, and high triglycerides levels are the major lipid components among patients with insulin resistance. Several non-statin agents, for example fibrates, are known to effectively improve these lipid abnormalities compared with statins. As for fibrates, RCTs, for example FIELD [6] or ACCORD [7], did not achieve good results for primary end point. The panels of the ACC/ AHA guideline, therefore, did not positively recommend fibrate usage. However, failure for the primary endpoint of these trials is probably because of the study design and the inclusion criteria for lipid abnormality. Sub-analysis of FIELD and ACCORD which examined results for high TG and/or low HDL-C patients, who were to be administered fibrates daily in a clinical setting, revealed the benefit of fibrate usage [7, 8]. Although the 2015 ADA guideline does not clearly recommend use of fibrates, it does not exclude their use, because the sub-analysis results from the ACCORD trial were referred to in the text.

After recognition of statins' supreme benefit in the early 2000s, from the perspective of ethics inclusion of statins in the trial protocol became almost obligate when designing the trial. Thus, when we evaluate recent nonstatin RCTs, we must take into account that the genuine effect of the agents examined might be obscured by the effect of the statins. Taking this into consideration, it might be necessary to include and evaluate previous RCTs, for example VA-HIT [9], even though their evidence levels were deemed intermediate. Notably, despite this circumstance, the recently published IMPROVE-IT trial clarified the benefit of ezetimibe added to statins, and the diabetic patients in IMPROVE-IT gained greater benefit than the whole cohort [10]. For the next revision of the guideline, ezetimibe should be included as a positive agent; however, other agents might also need to be re-investigated.

\section{Future perspective}

The issue of the 2011 report of the Institute of Medicine on the development of trustworthy clinical guidelines [4] is a great advance for the construction of guidelines, and it is absolutely necessary to utilize the results of high-quality evidence in constructing guidelines. However, at the same time, we must always consider whether or not the critical questions raised are adequate.

To achieve perfect guidelines is impossible; however, the guidelines, even if they are not perfect, should at least bring the greatest benefit to the patients and result in the best usability or convenience, even for non-specialists. Furthermore, the guidelines should vary depending on race, social factors, changes in disease structure, etc. There is not as much evidence appropriate for Japanese as for Caucasians; however, the Japan Atherosclerosis Society (JAS) guideline, which the Japan Diabetes Society (JDS) guideline follows for management of dyslipidemia, has been settled, taking into account much domestic and foreign evidence. It is thus, reasonable to use the JAS or JDS guidelines [11, 12] for management of dyslipidemia for Japanese diabetic patients.

Humans, unlike computers, possess wisdom. We have the ability to interpret results of trials by taking the study background into account, and to harmonize and combine several trial results. For settlement of sophisticated guidelines, we may need to utilize both wisdom and high-quality evidence.

Acknowledgments The author is extremely grateful to Dr Tamio Teramoto (Teikyo Academic Research Center, Teikyo University, Tokyo, Japan) for critical reading of this manuscript.

\section{Compliance with ethical standards}

Conflict of Interest The author declares that there is no conflict of interest.

Human rights statement and informed consent This article does not contain any studies with human or animal subjects performed by the author. 


\section{References}

1. Standards of medical care in diabetes-2015: summary of revisions. Diabetes care. 2015;38 Suppl 1:S4.

2. American Diabetes A. (8) Cardiovascular disease and risk management. Diabetes care. 2015;38 Suppl:S49-57.

3. Stone NJ, Robinson JG, Lichtenstein AH, Bairey Merz CN, Blum $\mathrm{CB}$, Eckel RH, et al. ACC/AHA guideline on the treatment of blood cholesterol to reduce atherosclerotic cardiovascular risk in adults: a report of the American College of Cardiology/American Heart Association Task Force on Practice Guidelines. Circulation. 2013;2014(129):S1-45.

4. Graham R, Mancher M, Wolman DM, Greenfield S, Steinberg E. Clinical practice guidelines we can trust. Washington, DC: National Academy of Sciences; 2011.

5. Boekholdt SM, Hovingh GK, Mora S, Arsenault BJ, Amarenco P, Pedersen TR, et al. Very low levels of atherogenic lipoproteins and the risk for cardiovascular events: a meta-analysis of statin trials. J Am Coll Cardiol. 2014;64:485-94.

6. Keech A, Simes RJ, Barter P, Best J, Scott R, Taskinen MR, et al. Effects of long-term fenofibrate therapy on cardiovascular events in 9795 people with type 2 diabetes mellitus (the FIELD study): randomised controlled trial. Lancet. 2005;366:1849-61.

7. The ACCORD Study Group. Effects of combination lipid therapy in type 2 diabetes mellitus. N Engl J Med. 2010;362:1563-74.
8. Scott R, O'Brien R, Fulcher G, Pardy C, D'Emden M, Tse D, et al. Effects of fenofibrate treatment on cardiovascular disease risk in 9,795 individuals with type 2 diabetes and various components of the metabolic syndrome: the Fenofibrate Intervention and Event Lowering in Diabetes (FIELD) study. Diabetes Care. 2009;32:493-8.

9. Rubins HB, Robins SJ, Collins D, Fye CL, Anderson JW, Elam $\mathrm{MB}$, et al. Gemfibrozil for the secondary prevention of coronary heart disease in men with low levels of high-density lipoprotein cholesterol. Veterans Affairs High-Density Lipoprotein Cholesterol Intervention Trial Study Group. $\mathrm{N}$ Engl $\mathrm{J}$ Med. 1999;341:410-8.

10. Cannon CP, Blazing MA, Giugliano RP, McCagg A, White JA, Theroux $P$, et al. Ezetimibe added to statin therapy after acute coronary syndromes. N Engl J Med. 2015;372:2387-97.

11. Teramoto T, Sasaki J, Ishibashi S, Birou S, Daida H, Dohi S, et al. Executive summary of the Japan Atherosclerosis Society (JAS) guidelines for the diagnosis and prevention of atherosclerotic cardiovascular diseases in Japan-2012 version. J Atheroscler Thromb. 2012;20:517-23.

12. Treatment Guide for Diabetes (2012-2013). In: Society JD, (ed) BUNKODO: http://www.jds.or.jp/common/fckeditor/editor/file manager/connectors/php/transfer.php?file=/uid000025_54726561 746D656E745F47756964655F666F725F44696162657465735F3 $23031322 \mathrm{D} 323031332 \mathrm{E} 706466$. 